Supporting information for:

\title{
Development, optimization, and evaluation of a duplex droplet digital PCR assay to quantify the $T$-nos/hmg copy number ratio in genetically modified maize
}

\author{
Félix-Urquídez Dalmira ${ }^{1,2}$, Pérez-Urquiza Melina ${ }^{2 *}$, León-Félix Josefina ${ }^{{ }^{*}}$, Valdez-Torres José \\ Benigno $^{1}$, García-Estrada Raymundo ${ }^{1}$, Acatzi-Silva Abraham ${ }^{3}$ \\ ${ }^{1}$ Center for Alimentation and Development Investigation, Culiacán, Sinaloa, México \\ ${ }^{2}$ National Metrology Center, El Marqués, Querétaro, México \\ ${ }^{3}$ Reference National Center for Detection of Genetically Modified Organisms, Tecámac, Estado de México, \\ México \\ *Corresponding authors: \\ meperez@cenam.mx \\ ljosefina@ciad.mx
}

\section{Table of contents}

Table S1. Primers and probes sequences used in the $T$-nos and $h m g$ amplification ...................S2 Table S2. Central composite design for optimization of a duplex ddPCR assay. DMR 447 Ila:

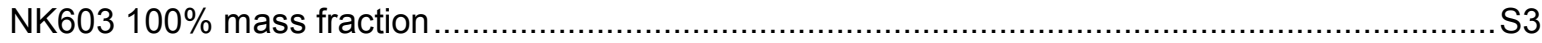

Table S3. Central composite design for optimization of a duplex ddPCR assay. DMR 447 Va:

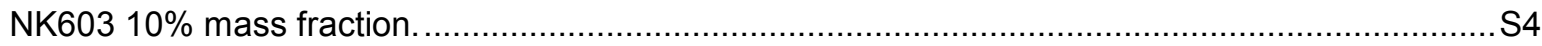

Table S4. Linear regression models built for optimized response variables in a duplex ddPCR method for $T$-nos/hmg analysis. DMR $447 \mathrm{lla}$ (100\% T-nos/hmg)......

Table S5. Linear regression models built for optimized response variables in a duplex ddPCR method for T-nos/hmg analysis. DMR $447 \mathrm{Va:} 10 \% \mathrm{~T}$-nos/hmg

Table S6. T-nos copy number, T-nos/hmg copy number ratio and relative standard deviation at different annealing temperatures.

Table S7.Average values and variability for $h m g c p$ at different $h m g$ primer/probe concentrations.S7

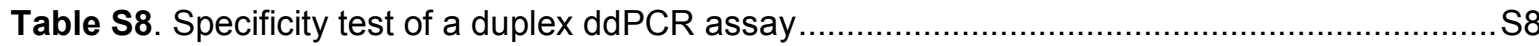

Table S9. Applicability assay of the $T$-nos/hmg ddPCR method, on several corn varieties............S9

Table S10. Comparison of quantification using singleplex and duplex ddPCR assays..............S9

Table S11. DNA restriction enzyme digestion. DMR 447 Va (10\% $T$-nos/hmg)

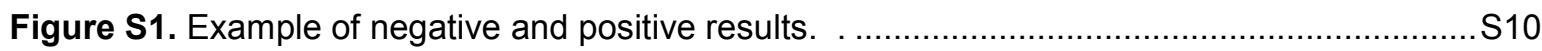

Figure S2. Overlaid contour plots for ddPCR optimization................................................... 11

Figure S3. DNA enzymatic restriction digestion....................................................................... 11

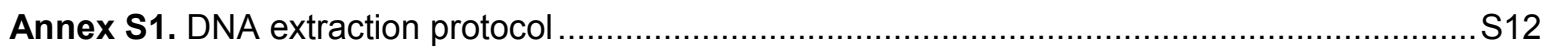

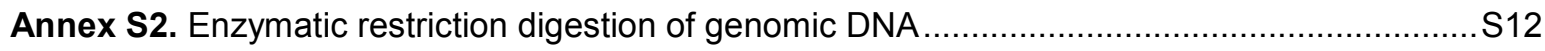

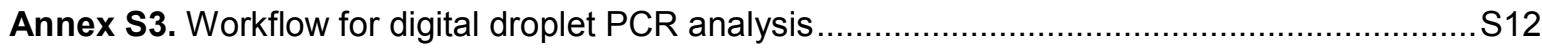


Table S1. Primers and probes sequences used in the $T$-nos and $h m g$ amplification

\begin{tabular}{|c|c|c|c|}
\hline Name & Sequence & $\begin{array}{c}\text { Amplicon } \\
\text { lenght }\end{array}$ & Reference \\
\hline Fw T-nos & 5'-CATGTAATGCATGACGTTATTTATG-3' & \multirow{4}{*}{$84 \mathrm{bp}$} & \multirow{4}{*}{$\begin{array}{l}\text { Reiting et al., } \\
2007\end{array}$} \\
\hline Rv T-nos & 5'-TTGTTTTTCTATCGCGTATTAAATGT-3' & & \\
\hline \multirow[t]{2}{*}{ T-nos probe } & 6FAM-ATGGGTTTTTATGATTAGAGTCCCGCAA- & & \\
\hline & MGBNFQ & & \\
\hline Fw hmg & 5'-TTGGACTAGAAATCTCGTGCTGA-3' & \multirow{3}{*}{79 bp } & \\
\hline Rv hmg & 5'-GCTACATAGGGAGCCTTGTCCT-3' & & \\
\hline hmg probe & VIC-CAATCCACACAAACGCACGCGTA-MGBNFQ & & \\
\hline
\end{tabular}


Table S2. Central composite design for optimization of a duplex ddPCR assay. DMR 447 Ila: NK603 100\% mass fraction

\begin{tabular}{|c|c|c|c|c|c|c|c|c|c|}
\hline \multirow[b]{2}{*}{ Order } & \multirow[b]{2}{*}{ Run } & \multirow[b]{2}{*}{$\mathrm{Pt}$} & \multirow[b]{2}{*}{ Block } & \multicolumn{3}{|c|}{ Coded values } & \multicolumn{3}{|c|}{ Natural values } \\
\hline & & & & T-nos & $\mathrm{Hmg}$ & $\mathrm{Ta}$ & $\begin{array}{l}\text { Tnos } \\
\text { (nM) }\end{array}$ & $\begin{array}{l}h m g \\
(\mathrm{nM})\end{array}$ & $\begin{array}{c}\mathrm{Ta} \\
\left({ }^{\circ} \mathrm{C}\right)\end{array}$ \\
\hline 10 & 1 & -1 & 1 & 1.682 & 0.000 & 0.000 & $\begin{array}{l}1002 \\
/ 384\end{array}$ & $\begin{array}{l}60 / \\
110\end{array}$ & 60 \\
\hline 18 & 2 & 0 & 1 & 0.000 & 0.000 & 0.000 & $\begin{array}{c}750 / \\
300\end{array}$ & $\begin{array}{l}60 / \\
110\end{array}$ & 60 \\
\hline 6 & 3 & 1 & 1 & 1.000 & -1.000 & 1.000 & $\begin{array}{c}900 / \\
350\end{array}$ & $\begin{array}{c}40 / \\
80\end{array}$ & 61 \\
\hline 20 & 4 & 0 & 1 & 0.000 & 0.000 & 0.000 & $\begin{array}{c}750 / \\
300\end{array}$ & $\begin{array}{l}60 / \\
110\end{array}$ & 60 \\
\hline 13 & 5 & -1 & 1 & 0.000 & 0.000 & -1.682 & $\begin{array}{c}750 / \\
300\end{array}$ & $\begin{array}{l}60 / \\
110\end{array}$ & 58 \\
\hline 4 & 6 & 1 & 1 & 1.000 & 1.000 & -1.000 & $\begin{array}{c}900 / \\
350\end{array}$ & $\begin{array}{l}80 / \\
140\end{array}$ & 59 \\
\hline 9 & 7 & -1 & 1 & -1.682 & 0.000 & 0.000 & $\begin{array}{c}498 / \\
216\end{array}$ & $\begin{array}{l}60 / \\
110\end{array}$ & 60 \\
\hline 7 & 8 & 1 & 1 & -1.000 & 1.000 & 1.000 & $\begin{array}{l}600 / \\
250\end{array}$ & $\begin{array}{l}80 / \\
140\end{array}$ & 61 \\
\hline 8 & 9 & 1 & 1 & 1.000 & 1.000 & 1.000 & $\begin{array}{c}900 / \\
350\end{array}$ & $\begin{array}{l}80 / \\
140\end{array}$ & 61 \\
\hline 14 & 10 & -1 & 1 & 0.000 & 0.000 & 1.682 & $\begin{array}{c}750 / \\
300\end{array}$ & $\begin{array}{l}60 / \\
110\end{array}$ & 62 \\
\hline 3 & 11 & 1 & 1 & -1.000 & 1.000 & -1.000 & $\begin{array}{c}600 / \\
250\end{array}$ & $\begin{array}{l}80 / \\
140\end{array}$ & 59 \\
\hline 17 & 12 & 0 & 1 & 0.000 & 0.000 & 0.000 & $\begin{array}{c}750 / \\
300\end{array}$ & $\begin{array}{l}60 / \\
110\end{array}$ & 60 \\
\hline 19 & 13 & 0 & 1 & 0.000 & 0.000 & 0.000 & $\begin{array}{c}750 / \\
300\end{array}$ & $\begin{array}{l}60 / \\
110\end{array}$ & 60 \\
\hline 12 & 14 & -1 & 1 & 0.000 & 1.682 & 0.000 & $\begin{array}{c}750 / \\
300\end{array}$ & $\begin{array}{c}95 / 16 \\
0\end{array}$ & 60 \\
\hline 2 & 15 & 1 & 1 & 1.000 & -1.000 & -1.000 & $\begin{array}{c}900 / \\
350\end{array}$ & $\begin{array}{c}40 / \\
80\end{array}$ & 59 \\
\hline 1 & 16 & 1 & 1 & -1.000 & -1.000 & -1.000 & $\begin{array}{c}600 / \\
250\end{array}$ & $\begin{array}{c}40 / \\
80\end{array}$ & 59 \\
\hline 16 & 17 & 0 & 1 & 0.000 & 0.000 & 0.000 & $\begin{array}{c}750 / \\
300\end{array}$ & $\begin{array}{l}60 / \\
110\end{array}$ & 60 \\
\hline 5 & 18 & 1 & 1 & -1.000 & -1.000 & 1.000 & $\begin{array}{c}600 / \\
250\end{array}$ & $\begin{array}{c}40 / \\
80\end{array}$ & 61 \\
\hline 11 & 19 & -1 & 1 & 0.000 & -1.682 & 0.000 & $\begin{array}{c}750 / \\
300\end{array}$ & $\begin{array}{c}25 / \\
60\end{array}$ & 60 \\
\hline 15 & 20 & 0 & 1 & 0.000 & 0.000 & 0.000 & $\begin{array}{c}750 / \\
300\end{array}$ & $\begin{array}{l}60 / \\
110\end{array}$ & 60 \\
\hline
\end{tabular}

Pt: point type; T-nos: $T$-nos primer/probe concentration; $h m g$ : $h m g$ primer/probe concentration; Ta: annealing temperature. Alpha $=1.68$ 
Table S3. Central composite design for optimization of a duplex ddPCR assay. DMR 447 Va: NK603 10\% mass fraction.

\begin{tabular}{|c|c|c|c|c|c|c|c|c|c|}
\hline \multirow[b]{2}{*}{ Order } & \multirow[b]{2}{*}{ Run } & \multirow[b]{2}{*}{ Pt } & \multirow[b]{2}{*}{ Block } & \multicolumn{3}{|c|}{ Coded values } & \multicolumn{3}{|c|}{ Natural values } \\
\hline & & & & T-nos & $\mathrm{Hmg}$ & $\mathrm{Ta}$ & $\begin{array}{l}\text { Tnos } \\
\text { (nM) }\end{array}$ & $\begin{array}{l}h m g \\
(\mathrm{nM})\end{array}$ & $\operatorname{Ta}\left({ }^{\circ} \mathrm{C}\right)$ \\
\hline 15 & 1 & 0 & 2 & 0.000 & 0.000 & 0.000 & $\begin{array}{c}750 / \\
300\end{array}$ & $\begin{array}{l}60 / \\
110\end{array}$ & 60 \\
\hline 9 & 2 & -1 & 2 & -1.682 & 0.000 & 0.000 & $\begin{array}{c}498 / \\
216\end{array}$ & $\begin{array}{l}60 / \\
110\end{array}$ & 60 \\
\hline 7 & 3 & 1 & 2 & -1.000 & 1.000 & 1.000 & $\begin{array}{c}600 / \\
250\end{array}$ & $\begin{array}{l}80 / \\
140\end{array}$ & 61 \\
\hline 20 & 4 & 0 & 2 & 0.000 & 0.000 & 0.000 & $\begin{array}{c}750 / \\
300\end{array}$ & $\begin{array}{l}60 / \\
110\end{array}$ & 60 \\
\hline 19 & 5 & 0 & 2 & 0.000 & 0.000 & 0.000 & $\begin{array}{c}750 / \\
300\end{array}$ & $\begin{array}{l}60 / \\
110\end{array}$ & 60 \\
\hline 6 & 6 & 1 & 2 & 1.000 & -1.000 & 1.000 & $\begin{array}{c}900 / \\
350\end{array}$ & $\begin{array}{c}40 / \\
80\end{array}$ & 61 \\
\hline 12 & 7 & -1 & 2 & 0.000 & 1.682 & 0.000 & $\begin{array}{c}750 / \\
300\end{array}$ & $\begin{array}{c}95 / 16 \\
0\end{array}$ & 60 \\
\hline 16 & 8 & 0 & 2 & 0.000 & 0.000 & 0.000 & $\begin{array}{c}750 / \\
300\end{array}$ & $\begin{array}{l}60 / \\
110\end{array}$ & 60 \\
\hline 4 & 9 & 1 & 2 & 1.000 & 1.000 & -1.000 & $\begin{array}{c}900 / \\
350\end{array}$ & $\begin{array}{l}80 / \\
140\end{array}$ & 59 \\
\hline 13 & 10 & -1 & 2 & 0.000 & 0.000 & -1.682 & $\begin{array}{c}750 / \\
300\end{array}$ & $\begin{array}{l}60 / \\
110\end{array}$ & 58 \\
\hline 3 & 11 & 1 & 2 & -1.000 & 1.000 & -1.000 & $\begin{array}{c}600 / \\
250\end{array}$ & $\begin{array}{l}80 / \\
140\end{array}$ & 59 \\
\hline 18 & 12 & 0 & 2 & 0.000 & 0.000 & 0.000 & $\begin{array}{c}750 / \\
300\end{array}$ & $\begin{array}{l}60 / \\
110\end{array}$ & 60 \\
\hline 1 & 13 & 1 & 2 & -1.000 & -1.000 & -1.000 & $\begin{array}{c}600 / \\
250\end{array}$ & $\begin{array}{c}40 / \\
80\end{array}$ & 59 \\
\hline 8 & 14 & 1 & 2 & 1.000 & 1.000 & 1.000 & $\begin{array}{c}900 / \\
350\end{array}$ & $\begin{array}{l}80 / \\
140\end{array}$ & 61 \\
\hline 11 & 15 & -1 & 2 & 0.000 & -1.682 & 0.000 & $\begin{array}{c}750 / \\
300\end{array}$ & $\begin{array}{c}25 / \\
60\end{array}$ & 60 \\
\hline 14 & 16 & -1 & 2 & 0.000 & 0.000 & 1.682 & $\begin{array}{c}750 / \\
300\end{array}$ & $\begin{array}{l}60 / \\
110\end{array}$ & 62 \\
\hline 2 & 17 & 1 & 2 & 1.000 & -1.000 & -1.000 & $\begin{array}{c}900 / \\
350\end{array}$ & $\begin{array}{c}40 / \\
80\end{array}$ & 59 \\
\hline 5 & 18 & 1 & 2 & -1.000 & -1.000 & 1.000 & $\begin{array}{c}600 / \\
250\end{array}$ & $\begin{array}{c}40 / \\
80\end{array}$ & 61 \\
\hline 7 & 19 & 0 & 2 & 0.000 & 0.000 & 0.000 & $\begin{array}{c}750 / \\
300\end{array}$ & $\begin{array}{l}60 / \\
110\end{array}$ & 60 \\
\hline 10 & 20 & -1 & 2 & 1.682 & 0.000 & 0.000 & $\begin{array}{l}1002 \\
/ 384\end{array}$ & $\begin{array}{l}60 / \\
110\end{array}$ & 60 \\
\hline
\end{tabular}

Pt: point type; T-nos: T-nos primer/probe concentration; $h m g$ : $h m g$ primer/probe concentration; Ta: annealing temperature. Alpha $=1.68$ 
Table S4. Linear regression models built for optimized response variables in a duplex ddPCR method for T-nos/hmg analysis. DMR 447 Ila (100\% T-nos/hmg).

\begin{tabular}{|c|c|c|c|c|}
\hline $\begin{array}{l}\text { Response } \\
\text { variable }\end{array}$ & Coefficient & $\begin{array}{c}p \\
\text { value }\end{array}$ & Regression model & $\begin{array}{c}\text { Adjusted } \\
\mathbf{R}^{2}\end{array}$ \\
\hline \multicolumn{5}{|l|}{$T$-nos/hmg } \\
\hline Constant & 52.53 & 0.000 & \multirow{3}{*}{$T-n o s / h m g=52.53-30.99 T a$} & \multirow{3}{*}{$70.34 \%$} \\
\hline $\mathrm{Ta}$ & -30.99 & 0.000 & & \\
\hline Lack of fit & - & 0.828 & & \\
\hline \multicolumn{5}{|l|}{$h m g c p$} \\
\hline Constant & 21,100 & 0.000 & \multirow[t]{2}{*}{ cp hmg = 21,100 } & \multirow[t]{2}{*}{-} \\
\hline Lack of fit & - & 0.580 & & \\
\hline \multicolumn{5}{|l|}{$T-\operatorname{nos} \Delta \mathrm{F}$} \\
\hline Constant & 3694.5 & 0.000 & \multirow{5}{*}{$\begin{array}{c}\Delta \mathrm{F} T-n o s=3694.5+862.7 T-n o s-2428.5 T a \\
+443.8 T a \times T a\end{array}$} & \multirow{5}{*}{$90.69 \%$} \\
\hline$T$-nos & 862.7 & 0.000 & & \\
\hline$T a$ & -2428.5 & 0.000 & & \\
\hline TaxTa & 443.8 & 0.028 & & \\
\hline Lack of fit & - & 0.024 & & \\
\hline \multicolumn{5}{|l|}{$h m g \Delta \mathrm{F}$} \\
\hline Constant & 995.75 & 0.000 & \multirow{5}{*}{$\begin{array}{c}\Delta \mathrm{F} h m g=995.8+432.0 \mathrm{hmg}-63.4 \mathrm{Ta}+ \\
45.4 \mathrm{hmgxhmg}\end{array}$} & \multirow{5}{*}{$94.85 \%$} \\
\hline$h m g$ & 432.02 & 0.000 & & \\
\hline $\mathrm{Ta}$ & -63.43 & 0.015 & & \\
\hline hmgxhmg & 45.39 & 0.061 & & \\
\hline Lack of fit & - & 0.981 & & \\
\hline
\end{tabular}

T-nos/hmg: T-nos/hmg copy number ratio. $\boldsymbol{h m g} \boldsymbol{c p}: \mathbf{h m g}$ copy number. $T$-nos $\Delta \mathrm{F}$ : Fluorescence difference between $T$-nos positive and $T$-nos negative droplets. $h m g \Delta \mathrm{F}$ : Fluorescence difference between $h m g$ positive and $h m g$ negative droplets. T-nos: T-nos primer/probe concentration. $\mathbf{h m g}$ : $\mathbf{h m g}$ primer/probe concentration. $\mathbf{T a}$ : annealing temperature. ddPCR: digital droplet polymerase chain reaction. $\mathbf{R}^{2}$ : coefficient of determination. DMR 447 Ila: certified reference material for NK603 (100\% mass fraction). 
Table S5. Linear regression models built for optimized response variables in a duplex ddPCR method for T-nos/hmg analysis. DMR 447 Va: $10 \% T$-nos/hmg

\begin{tabular}{|c|c|c|c|c|}
\hline $\begin{array}{c}\text { Response } \\
\text { variable }\end{array}$ & Coefficient & $\begin{array}{c}\mathbf{p} \\
\text { value }\end{array}$ & Regression model & $\begin{array}{c}\text { Adjusted } \\
\mathbf{R}^{2} \\
\end{array}$ \\
\hline \multicolumn{5}{|l|}{ T-nos/hmg } \\
\hline Constant & 5.06 & 0.000 & & \\
\hline$T$-nos & 0.76 & 0.060 & $T-n o s / h m g=5.06+0.76 T-\operatorname{nos}-2.58 T a$ & $71.71 \%$ \\
\hline $\mathrm{Ta}$ & -2.58 & 0.000 & & \\
\hline Lack of fit & - & 0.504 & & \\
\hline \multicolumn{5}{|l|}{$h m g c p$} \\
\hline Constant & 21968.3 & 0.000 & & \\
\hline$h m g$ & -876.6 & 0.066 & cp hmg $=21968.3-876.6 h m g$ & $13.02 \%$ \\
\hline Lack of fit & - & 0.872 & & \\
\hline \multicolumn{5}{|l|}{$T-\operatorname{nos} \Delta \mathrm{F}$} \\
\hline Constant & 3483.77 & 0.000 & & \\
\hline$T$-nos & 805.92 & 0.000 & & \\
\hline$h m g$ & 29.41 & 0.864 & $\Delta \mathrm{F} T$-nos $=3483.8+805.9 T-n o s-2546.5 T a+$ & \\
\hline$T a$ & -2546.45 & 0.000 & 394.6hmgxhmg -759.6T-nosxTa + & $93.43 \%$ \\
\hline$h m g x h m g$ & 394.56 & 0.030 & $512.2 \mathrm{hmgxTa}$ & \\
\hline$T-n o s x T a$ & -759.63 & 0.004 & & \\
\hline hmgxTa & 512.23 & 0.036 & & \\
\hline Lack of fit & - & 0.693 & & \\
\hline \multicolumn{5}{|l|}{$h m g \Delta \mathrm{F}$} \\
\hline Constane & 1086.5 & 0.000 & & \\
\hline$h m g$ & 445.6 & 0.000 & $\Delta \mathrm{F} h m g=1086.5+445.6 h m g-125.7 T a$ & $95.16 \%$ \\
\hline$T a$ & -125.7 & 0.000 & & \\
\hline Lack of fit & - & 0.234 & & \\
\hline
\end{tabular}

T-nos/hmg: $T$-nos/hmg copy number ratio. $h m g ~ c p: ~ h m g$ copy number. $T$-nos $\Delta \mathrm{F}$ : Fluorescence difference between $T$-nos positive and $T$-nos negative droplets. $h m g$ F : Fluorescence difference between $h m g$ positive and $h m g$ negative droplets. T-nos: T-nos primer/probe concentration. $\mathbf{h m g}$ : $\mathbf{h m g}$ primer/probe concentration. Ta: annealing temperature. ddPCR: digital droplet polymerase chain reaction. $\mathbf{R}^{2}$ : coefficient of determination. DMR 447 Va: certified reference material for NK603 (10\% mass fraction). 
Table S6. T-nos copy number, $T$-nos/hmg copy number ratio and relative standard deviation at different annealing temperatures.

\begin{tabular}{|c|c|c|c|c|c|c|}
\hline \multirow{2}{*}{$\begin{array}{l}\text { Annealing } \\
\text { temperature }\end{array}$} & \multicolumn{2}{|c|}{$T-\operatorname{nos} c p$} & \multicolumn{2}{|c|}{$\begin{array}{c}T \text {-nos/hmg copy } \\
\text { number ratio }\end{array}$} & \multicolumn{2}{|c|}{$\begin{array}{l}\text { RSD for } T \text {-nos/hmg } \\
\text { copy number ratio }\end{array}$} \\
\hline & DMR IIa & DMR Va & DMR IIa & DMR Va & DMR Ila & DMR Va \\
\hline $58^{\circ} \mathrm{C}$ & 17,207 & 1747 & $90.5 \%$ & $8.0 \%$ & $0.1 \%$ & $0.2 \%$ \\
\hline $59^{\circ} \mathrm{C}$ & 18,334 & 1715 & $87.9 \%$ & $7.8 \%$ & $1.2 \%$ & $5.4 \%$ \\
\hline $60^{\circ} \mathrm{C}$ & 10,579 & 1193 & $52.8 \%$ & $5.2 \%$ & $40.6 \%$ & $37.7 \%$ \\
\hline $61^{\circ} \mathrm{C}$ & 3,802 & 506 & $20.1 \%$ & $2.4 \%$ & $76.2 \%$ & $39.6 \%$ \\
\hline $62^{\circ} \mathrm{C}$ & 10 & 0 & $0.1 \%$ & $0.0 \%$ & $0.1 \%$ & $0.0 \%$ \\
\hline
\end{tabular}

Average values at every temperature level. DMR Ila: NK603 (100\% mass fraction). DMR Va: (10\% mass fraction). cp: copies. RSD: relative standard deviation.

Table S7. Average values and variability for $h m g c p$ at different $h m g$ primer/probe concentrations.

\begin{tabular}{cccccc}
\hline \multicolumn{2}{c}{\begin{tabular}{c}
\multicolumn{2}{c}{$\boldsymbol{h} \boldsymbol{c}$ concentration } \\
\multicolumn{2}{c}{ primer/probe }
\end{tabular}} & \multicolumn{2}{c}{ RSD for $\boldsymbol{h m g} \boldsymbol{c p}$} \\
\hline \multirow{2}{*}{ Coded level } & Natural level & DMR 447 lla & DMR 447 & DMR 447 & DMR 447 \\
& & & Va & Ila & Va \\
$\mathbf{- 1 . 6 8}$ & $25 / 40 \mathrm{nM}$ & 18890 & 24,663 & $3 \%$ & $14 \%$ \\
$\mathbf{- 1 . 0}$ & $40 / 80 \mathrm{nM}$ & 20934 & 22,627 & $10 \%$ & $15 \%$ \\
$\mathbf{0}$ & $60 / 110 \mathrm{nM}$ & 21058 & 21,820 & $18 \%$ & $10 \%$ \\
$\mathbf{1 . 0}$ & $80 / 140 \mathrm{nM}$ & 19074 & 21,393 & $4 \%$ & $4 \%$ \\
$\mathbf{1 . 6 8}$ & $95 / 160 \mathrm{nM}$ & 21112 & 20,570 & $4 \%$ & $4 \%$ \\
\hline
\end{tabular}

Average values at every $\mathrm{hmg}$ primer/probe concentration level. Data obtained from optimization of a duplex ddPCR method for T-nos/hmg analysis. DMR 447 lla: 100\% mass fraction. DMR $447 \mathrm{Va:} 10 \%$ mass fraction. $\mathrm{cp}$ : copies. RSD: relative standard deviation. 
Table S8. Specificity test of a duplex ddPCR assay

\begin{tabular}{|c|c|c|c|c|c|}
\hline Species & Material & $\begin{array}{c}\text { Transformation } \\
\text { event }\end{array}$ & $\begin{array}{c}\text { Mass } \\
\text { fraction }\end{array}$ & T-nos & $h m g$ \\
\hline \multicolumn{6}{|l|}{ Maize } \\
\hline Popcorn & $*$ & --- & --- & - & + \\
\hline Red & $*$ & --- & --- & - & + \\
\hline Blue & $*$ & --- & --- & - & + \\
\hline White & $*$ & --- & --- & - & + \\
\hline \multirow[t]{9}{*}{ Yellow } & DMR 436 la & MON 810 & No GM & - & + \\
\hline & DMR 453 la & MON 88017 & No GM & - & + \\
\hline & DMR 451 la & MON 863 & No GM & - & + \\
\hline & DMR 482 la & --- & No GM & - & + \\
\hline & DMR 436 Vb & MON 810 & $10 \%$ & - & + \\
\hline & DMR 447 IIla & NK603 & $1 \%$ & + & + \\
\hline & DMR $451 \mathrm{Va}$ & MON 863 & $10 \%$ & + & + \\
\hline & DMR 452 Ila & MON 89034 & $100 \%$ & + & + \\
\hline & DMR $453 \mathrm{Va}$ & MON 88017 & $10 \%$ & + & + \\
\hline \multirow[t]{2}{*}{ Soybean } & DMR 495 Ila & MON 04032-6 & $100 \%$ & + & - \\
\hline & DMR 495 IIIa & MON 04032-6 & $1 \%$ & + & - \\
\hline \multirow[t]{2}{*}{ Wheat } & DMR 496 Ila & DREB-1 ${ }^{\mathrm{a}}$ & $100 \%$ & + & - \\
\hline & DMR 496 IIla & DREB-1ㅁa & $1 \%$ & + & - \\
\hline Rice & $*$ & --- & --- & - & - \\
\hline
\end{tabular}

* Materials acquired as seeds at local markets.

The sign showed in the $T$-nos result and $h m g$ result columns, specifies if there was (+) or not (-) amplification of the corresponding target. 
Table S9. Applicability assay of the T-nos/hmg ddPCR method, on several corn varieties.

\begin{tabular}{ccccc}
\hline $\begin{array}{c}\text { Tested } \\
\text { variety }\end{array}$ & $\begin{array}{c}\text { Expected T- } \\
\text { nos/hmg copy } \\
\text { number ratio }\end{array}$ & $\begin{array}{c}\text { Measured T- } \\
\text { nos/hmg copy } \\
\text { number ratio }\end{array}$ & $\begin{array}{c}\text { Relative } \\
\text { standard } \\
\text { deviation }\end{array}$ & Accuracy \\
\hline $\begin{array}{c}\text { Popping } \\
\text { corn }\end{array}$ & $0.91 \%$ & $1.0 \%$ & $5.0 \%$ & $111.43 \%$ \\
\hline Blue corn & $0.85 \%$ & $0.93 \%$ & $3.5 \%$ & $108.72 \%$ \\
& & & & \\
Red corn & $0.99 \%$ & $1.13 \%$ & $5.5 \%$ & $113.56 \%$ \\
\hline White corn & $0.84 \%$ & $0.90 \%$ & $1.2 \%$ & $108.08 \%$ \\
\hline
\end{tabular}

Average from three replicates. Tested samples were prepared as a mix of DNA extracted from non-GM corn and GM corn for every variety. The analyzed variety represented at least $97.5 \%$ of the final mix.

Table S10. Comparison of quantification using singleplex and duplex ddPCR assays

\begin{tabular}{|c|c|c|c|c|c|c|}
\hline Material & Measurand & Duplex (Dx) & $\begin{array}{c}\text { Singleplex } \\
\left(S_{x}\right)\end{array}$ & $\begin{array}{c}\% C V \\
\text { duplex }\end{array}$ & $\begin{array}{c}\% \text { CV } \\
\text { singleplex }\end{array}$ & $\begin{array}{l}\% \text { Bias } \\
D x / S x\end{array}$ \\
\hline Dilution & $T$-nos cp & 20 & 18 & 3.2 & 13.0 & 6.6 \\
\hline from DMR & hmg cp & 27819 & 28235 & 0.8 & 2.0 & -1.3 \\
\hline 447 IIIa & $T-n o s / h m g \%$ & 0.070 & 0.062 & 2.5 & 13.8 & 6.5 \\
\hline \multirow{3}{*}{$\begin{array}{c}\text { DMR } 447 \\
\text { Illa }\end{array}$} & $T$-nos cp & 185 & 174 & 5.9 & 7.8 & 6.3 \\
\hline & hmg cp & 20486 & 20872 & 1.1 & 0.9 & -1.8 \\
\hline & $T-n o s / h m g \%$ & 0.90 & 0.84 & 5.0 & 8.1 & 8.2 \\
\hline \multirow{3}{*}{ DMR $447 \mathrm{Va}$} & $T-n o s \mathrm{cp}$ & 1563 & 1675 & 2.4 & 1.7 & -6.7 \\
\hline & hmg cp & 20553 & 20630 & 1.9 & 1.7 & -0.4 \\
\hline & $T$-nos/hmg \% & 7.6 & 8.1 & 2.7 & 0.3 & -6.3 \\
\hline \multirow{3}{*}{ DMR 452 Ila } & $T-\operatorname{nos} \mathrm{cp}$ & 17001 & 17572 & 1.6 & 1.9 & -3.2 \\
\hline & hmg cp & 32023 & 32122 & 1.8 & 1.3 & -0.3 \\
\hline & $T$-nos/hmg \% & 53.1 & 54.7 & 1.8 & 1.2 & -2.9 \\
\hline \multirow{3}{*}{ DMR 447 Ila } & $T-n o s \mathrm{cp}$ & 14242 & 14069 & 4.0 & 0.3 & 1.2 \\
\hline & hmg cp & 16040 & 15819 & 4.5 & 1.6 & 1.4 \\
\hline & T-nos/hmg \% & 88.8 & 89.0 & 2.0 & 1.9 & -0.2 \\
\hline
\end{tabular}

Average from three replicates. *This material was prepared as a dilution from DMR 447 IIla (1\% mass fraction), so the final concentration was not the same for duplex and singleplex assay $(0.074$ and $0.07 \%$, respectively). To address this issue, the bias was estimated taking into account the accuracy achieved in every case. 
Table S11. DNA restriction enzyme digestion. DMR 447 Va (10\% T-nos $/ \mathrm{hmg})$

\begin{tabular}{cccccc}
\hline Measurand & $\begin{array}{c}\text { Non- } \\
\text { digested } \\
\text { DNA }\end{array}$ & $\begin{array}{c}\text { BamHI } \\
\text { digestion }\end{array}$ & $\begin{array}{c}\text { EcoRI } \\
\text { digestion }\end{array}$ & $\begin{array}{c}\text { Multiple } \\
\text { digestion }\end{array}$ & $\begin{array}{c}\text { Xhol } \\
\text { digestion }\end{array}$ \\
\hline T-nos cp & 1491 & 1739 & 1645 & 1625 & 1336 \\
hmg cp & 18895 & 17844 & 17398 & 17815 & 17552 \\
$T$-nos/hmg\% & $7.9^{\mathrm{bc}}$ & $9.8^{\mathrm{a}}$ & $9.5^{\mathrm{ab}}$ & $9.1^{\mathrm{abc}}$ & $7.6^{\mathrm{c}}$ \\
\hline
\end{tabular}

Average from three replicates. Treatments not sharing a letter are significantly different. (Tukey method, $\mathrm{P} \leq 0.05)$.
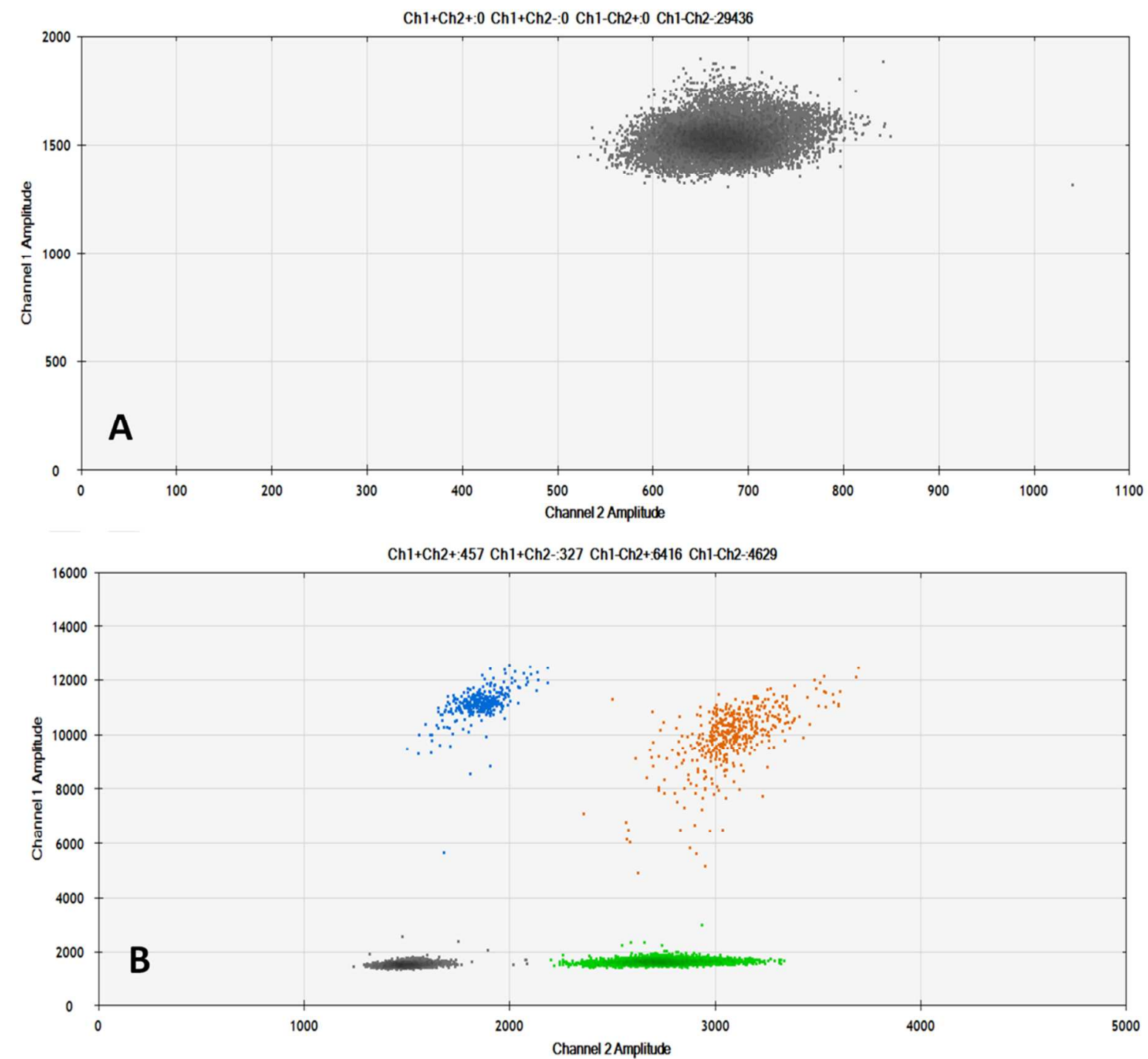

Figure S1. Example of negative and positive results. Results from a non-template control (A) and a genetically modified maize sample (B). Each point represents a droplet with a given fluorescence level. Droplet colors indicate which target was amplified: $T$-nos (blue), $h m g$ (green), none of the two (gray) or both (orange). 


\section{Contour plot. DMR 447 Ila (100\%)}

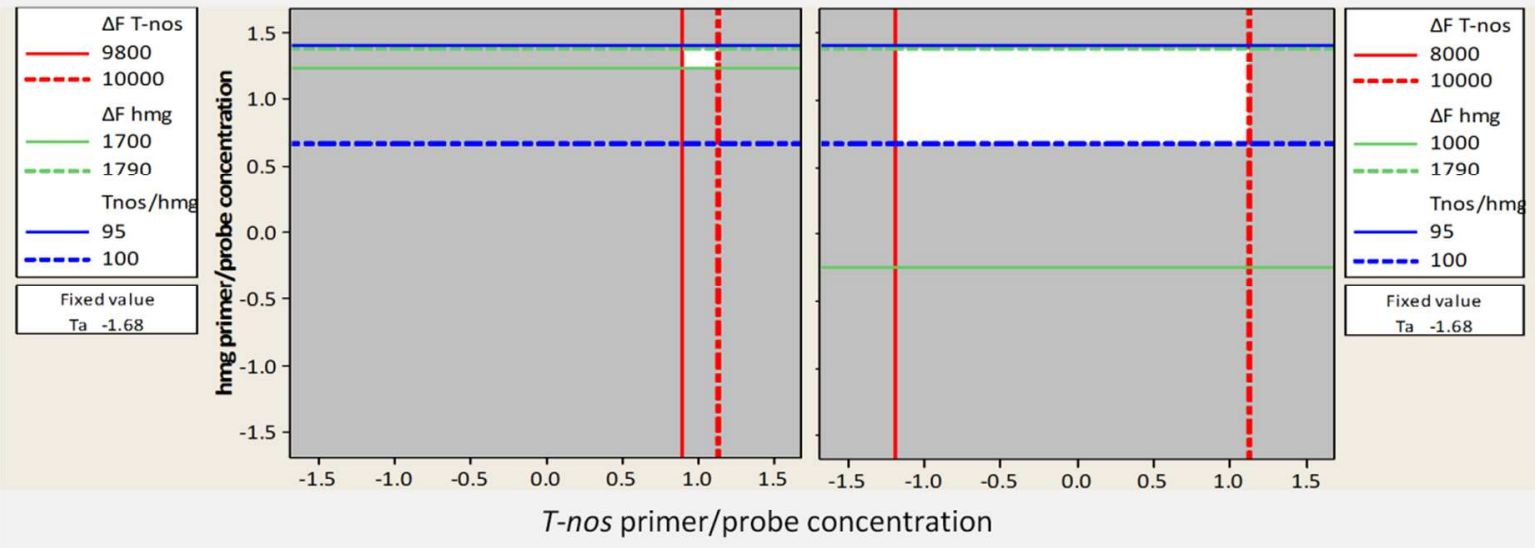

Contour plot. DMR 447 Va (10\%)

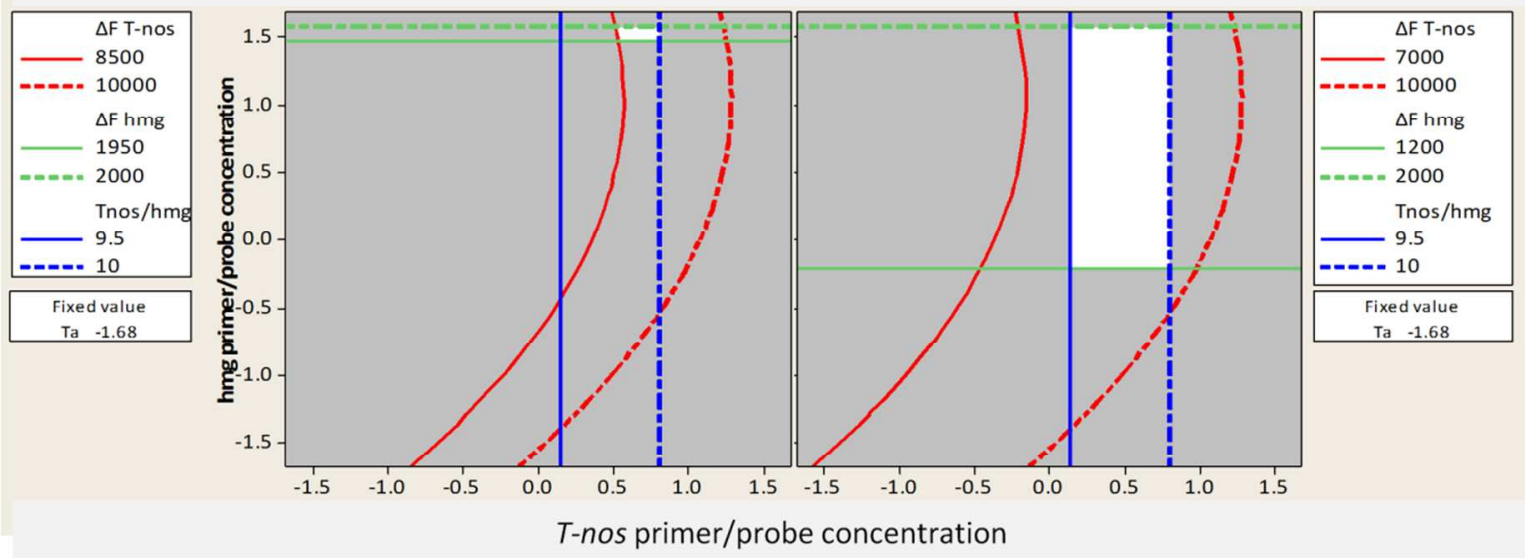

Figure S2. Overlaid contour plots for ddPCR optimization. 1) DMR 447 Ila (100\%). 2) DMR 447 Va $(10 \%)$. The white region shows the operating range, where the response variables are predicted to be the ones shown in the lateral boxes.

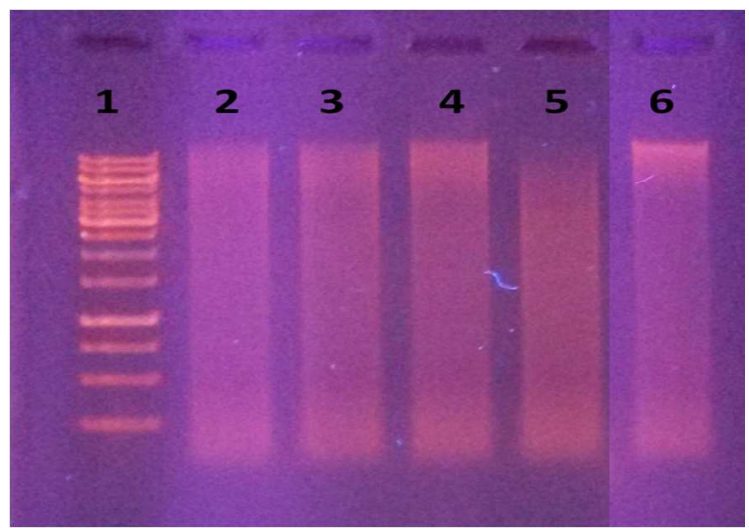

Figure S3. DNA enzymatic restriction digestion. 1) DNA ladder; 2) EcoRl; 3) BamHI; 4) Xhol; 5) Multiple digestion; 6) Non-digested DNA. 


\section{Annex S1. DNA extraction protocol}

The genomic DNA was extracted using the Genomic DNA extraction kit (FAST ID, USA). $500 \mu \mathrm{L}$ of Genomic Lyse buffer were added to $100 \mathrm{mg}$ of maize seed-powder flour, followed by $10 \mu \mathrm{L}$ of proteinasa $\mathrm{K}(10 \mathrm{mg} / \mathrm{mL})$. The sample was shacked on termomixer (Bioer, China) for $30 \mathrm{~min}\left(16^{\circ} \mathrm{C}\right.$; $1,500 \mathrm{rpm}$ ) and briefly on vortex at the end. It was centrifuged (labtron scientific, UK) for $12 \mathrm{~min}$ $\left(16^{\circ} \mathrm{C} ; 12,000 \mathrm{rpm}\right)$; the supernatant was collected and $400 \mu \mathrm{L}$ of Genomic Bind buffer were added. The sample was shaked on termomixer $\left(1 \mathrm{~min}, 20^{\circ} \mathrm{C}, 1,500 \mathrm{rpm}\right)$ and loaded on a DNA Binding Column. A centrifugal force $\left(5 \mathrm{~min} ; 16^{\circ} \mathrm{C} ; 10,000 \mathrm{rpm}\right)$ was applied to pass the sample through the column, and the eluates were discarded. $400 \mu \mathrm{L}$ of Genomic Wash Buffer were added to the column and it was centrifuged again for $5 \mathrm{~min}\left(16^{\circ} \mathrm{C} ; 10,000 \mathrm{rpm}\right)$, discarding the eluates. The column was washed three times with $400 \mu \mathrm{L}$ of $75 \%$ ethanol; at the end of every wash the column was centrifuged for $1 \mathrm{~min}\left(16^{\circ} \mathrm{C} ; 5,000 \mathrm{rpm}\right)$. The extraction column was transferred to a new vial and it was added with $100 \mu \mathrm{L}$ of Extraction buffer (1XTE). The samples were incubated for $10 \mathrm{~min}$ at $65^{\circ} \mathrm{C}$, and later centrifuged for $2 \min \left(16^{\circ} \mathrm{C} ; 5,000 \mathrm{rpm}\right)$. The eluted DNA was collected and stored at $-40^{\circ} \mathrm{C}$.

\section{Annex S2. Workflow for digital droplet PCR analysis}

A mix containing the following reagents was prepared: $10 \mu \mathrm{L}$ of supermaster mix (Bio-Rad, USA), 1 $\mu \mathrm{L}$ (or the volume needed according to starting and final concentrations desired) of $T$-nos and $h m g$ primers and probes, and the volume needed of water for a final volume of $19 \mu \mathrm{L}$. This solution was mixed with $1 \mu \mathrm{L}$ of template DNA. $20 \mu \mathrm{L}$ of this mix were placed on the sample wells of an 8-well cartridge; the oil wells were filled with $70 \mu \mathrm{L}$ of droplet generator oil. Droplets were generated using the QX200 droplet generator (Bio-Rad, USA). Water-in-oil emulsions were transferred to a 96-well plate and amplified in a GeneAmp 9500 PCR system (Applied BioSystems, USA). Thermal cycling conditions were: $15 \mathrm{~min}$ at $95^{\circ} \mathrm{C}$ for enzyme activation ( 1 cycle), followed by $15 \mathrm{~s}$ at $94^{\circ} \mathrm{C}$ for DNA denaturation, and $1 \mathrm{~min}$ at the temperature indicated for the experimental design (or the defined optimal temperature: $58^{\circ} \mathrm{C}$ ) for annealing and extension (45 cycles). Products were cooled to $4^{\circ} \mathrm{C}$. After amplification, plates were placed on the QX200 droplet reader (Bio-Rad, USA) for data acquisition. FAM signal ( $T$-nos) was registered in channel 1 , and VIC signal $(h m g)$ was measured in channel 2 . Only samples with a minimum of 8,000 accepted droplets were considered for subsequent analysis. 


\section{Annex S3. Enzymatic restriction digestion of genomic DNA}

For restriction digestion assay, a mix was prepared adding the following reagents in the order stated: $12 \mu \mathrm{L}$ of water (molecular biology grade), $2 \mu \mathrm{L}$ of 10X FastDigest buffer (Thermo scientific, USA), $4 \mu \mathrm{L}$ of DNA and $2 \mu \mathrm{L}$ of enzyme (Thermo scientific, USA); it was gently homogenized. The mix was incubated for $5 \mathrm{~min}(\mathrm{BamHI}), 10 \mathrm{~min}(\mathrm{Xhol})$ or $20 \mathrm{~min}$ (EcoRI and multiple digestion) in a bath water at $37^{\circ} \mathrm{C}$. This solution was used as template DNA for later droplet generation, amplification and analysis by ddPCR. 